Bull. Austral. Math. Soc.

VOL. 53 (1996) [235-247]

\title{
SEMILINEAR PROBLEMS ON THE HALF SPACE WITH A HOLE
}

\section{HWAI-CHIUAN WANG}

In this article we prove that there is a positive solution in $H_{0}^{1}(\Omega)$ of the equation $-\Delta u+\lambda u=|u|^{p-2} u$ in $\Omega$ where $\Omega$ is the half space with a hole, $\lambda>0$ and $2<p<2 N /(N-2)$.

\section{INTRODUCTION}

In this article we use the following notation:

$\mathrm{R}^{N}$ : the $N$-dimensional Euclidean space, $N \geqslant 3$, $\mathbf{R}_{+}^{N}=\left\{\left(x^{\prime}, x_{N}\right) \in \mathbf{R}^{N-1} \times \mathbf{R} \mid 0<x_{N}<\infty\right\}:$ the upper half space, $\mathbf{R}_{-}^{N}=\left\{\left(x^{\prime}, x_{N}\right) \in \mathbf{R}^{N-1} \times \mathbf{R} \mid-\infty<x_{N}<0\right\}:$ the lower half space, $\Omega_{r}$ an unbounded smooth domain such that $\bar{\Omega}_{r} \subset \mathbf{R}_{+}^{N}, a_{r}=(a, r) \notin \bar{\Omega}_{r}$, and its complement $\bar{\Omega}_{r}^{c}$ is contained in a ball $B_{\rho}\left(a_{r}\right)$ centred at $a_{r}$ with radius $\rho$ : the upper half space with a hole.

$\mathrm{D}$ : One of $\mathbf{R}^{N}, \mathbf{R}_{+}^{N}$ and $\Omega_{r}$.

For $\lambda>0$ and $2<p<2 N /(N-2)$, consider the semilinear elliptic equation:

$\left(1_{D}\right)$

$$
\left\{\begin{array}{l}
-\Delta u+\lambda u=|u|^{p-2} u \text { in } \mathrm{D} \\
u \in H_{0}^{1}(\mathrm{D})
\end{array}\right.
$$

$H_{0}^{1}(\mathrm{D})$ : the usual Sobolev space on D under the norm

$$
\|u\|_{D}^{2}=\int_{D}\left(|\nabla u|^{2}+\lambda u^{2}\right)
$$

For $u \in H_{0}^{1}(\mathrm{D})$, define

$$
\begin{aligned}
f_{D}(u) & =\int_{D}\left(|\nabla u|^{2}+\lambda u^{2}\right), \\
M_{D} & =\left\{\left.u \in H_{0}^{1}(D)\left|\int_{D}\right| u\right|^{p}=1\right\}, \\
\alpha_{D} & =\inf \left\{f_{D}(u) \mid u \in M_{D}\right\}, \\
F_{D}(u) & =\frac{1}{2} \int_{D}\left(|\nabla u|^{2}+\lambda u^{2}\right)-\frac{1}{p} \int_{D}|u|^{p} .
\end{aligned}
$$

Received 1st May, 1995

I would like to express my gratitude to the referee for his valuable suggestions.

Copyright Clearance Centre, Inc. Serial-fee code: 0004-9729/96 \$A2.00+0.00. 
Write $\|\cdot\|, f, M, \alpha, F$ for $\|\cdot\|_{\mathbf{R}^{N}}, f_{\mathbf{R}^{N}}, M_{\mathbf{R}^{N}}, \alpha_{\mathbf{R}^{N}}, F_{\mathbf{R}^{N}}$, respectively.

The motivation to study our problem is as follows: by applying the compactness of the embedding $H_{r}^{1}\left(\mathbf{R}^{N}\right) \hookrightarrow L^{P}\left(\mathbf{R}^{N}\right)$, where $H_{r}^{1}\left(\mathbf{R}^{N}\right)$ consists of the radially symmetric functions in $H^{1}\left(\mathbf{R}^{N}\right)$, Berestycki-Lions [4] proved that $\alpha$ is achieved, and hence concluded that there is a positive solution of equation $\left(1_{\mathbf{R}^{N}}\right)$. Gidas-Ni-Nirenberg [9] proved that every positive solution $u$ of equation $\left(1_{\mathbf{R}^{N}}\right)$ is radially symmetric with respect to some point in $\mathbf{R}^{N}$ satisfying

$$
\left\{\begin{array}{lll}
u(r) r^{(N-1) / 2} e^{\sqrt{\lambda} r}=\gamma+o(1) & \text { as } & r \rightarrow \infty \\
u^{\prime}(r) r^{(N-1) / 2} e^{\sqrt{\lambda} r}=-\sqrt{\lambda} \gamma+o(1) & \text { as } & r \rightarrow \infty
\end{array}\right.
$$

where $\gamma>0$ a constant. Kwong $[11]$ proved that the positive solution of $\left(1_{R^{N}}\right)$ is unique up to translations. Throughout this article denote by $\bar{u}$ the unique solution of equation $\left(1_{\mathbf{R}^{N}}\right)$ which attains its maximum at $0, \int_{\mathbf{R}^{N}}|\bar{u}|^{p}=1,\|\bar{u}\|^{2}=\alpha$, and satisfies (1-1).

Esteban-Lions [8] used the infinitesimal $\frac{\partial}{\partial y}$ of the translation operators to derive an important integral identity for the equation $-\Delta u=f(u)$ in an unbounded domain with boundary $\Gamma$ :

$$
\int_{\Gamma} n_{i}(x)|\nabla u|^{2} d s=0 \quad \text { for } \quad 1 \leqslant i \leqslant N
$$

Let $\Omega_{1}=\left\{\left(x^{\prime}, x_{N}\right) \in \mathbf{R}^{N-1} \times \mathbf{R}|| x^{\prime} \mid<1,0<x_{N}\right\}$ be an upper half strip. Two of its consequences are that there does not exist any nontrivial solution neither in $H_{0}^{1}\left(\mathbf{R}_{+}^{N}\right)$ of equation $\left(1_{R_{+}^{N}}\right)$ nor in $H_{0}^{1}\left(\Omega_{1}\right)$ of equation $\left(1_{\Omega_{1}}\right)$. Such a surprising result attracted mathematicians to study the equations on the half space $R_{+}^{N}$ and on $\Omega_{1}$. Ai-Zhu [1] proved that there are positive solutions of the equation

$$
\begin{cases}-\triangle u+\lambda u=|u|^{p-2} u & \text { in } \mathbf{R}_{+}^{N} \\ u>0 & \text { in } \mathbf{R}_{+}^{N} \\ u\left(x^{\prime}, 0\right)=f\left(x^{\prime}\right) & \text { on } \partial \mathbf{R}_{+}^{N},\end{cases}
$$

where $f \geqslant 0, f \not \equiv 0$ in $H^{1 / 2}\left(\mathbf{R}^{N-1}\right) \cap L^{\infty}\left(\mathbf{R}^{N-1}\right)$. In 1992, the author gave a talk in the second nonlinear France-Taiwan PDE Conference held in Paris. We proved that if $r$ is large, $\Omega_{2}=\Omega_{1} \cup B_{r}(0)$ the upper half strip adding a big ball, then there is a positive solution in $H_{0}^{1}\left(\Omega_{2}\right)$ of equation $\left(1_{\Omega_{2}}\right)$ (see Lien-Tzeng-Wang [12, Example 5.6, p.1296]). In my talk, Berestycki asked the following problem: is there any positive solution of the equation on the upper half strip with a hole? We have only partial result for the Berestycki problem. However in this article, we try to answer a related problem affirmatively: 
THEOREM A. There is $\rho_{0}>0$ and $r_{0}>0$ such that if $0<\rho \leqslant \rho_{0}$ and $r \geqslant r_{0}$ then there is a positive solution of equation $\left(1_{\Omega_{r}}\right)$.

To prove Theorem A we use a higher energy process through a barycentre function. Such a process was first used by Coron [7], then by Benci-Cerami [3], Grossi [10] and many others. In this article we adapt several tools from Benci-Cerami [3] and Grossi $[\mathbf{1 0}]$.

\section{Existence of SOLUTIONS}

For $c \in \mathbf{R}$, a $(P S)_{c}$-sequence in $H_{0}^{1}\left(\Omega_{r}\right)$ for $F$ is a sequence $\left\{u_{n}\right\}$ such that

$$
\begin{aligned}
F\left(u_{n}\right) & \longrightarrow c, \\
F^{\prime}\left(u_{n}\right) \longrightarrow 0 & \text { strongly in } H^{-1}\left(\Omega_{r}\right) .
\end{aligned}
$$

We state a classical and interesting known decomposition theorem for a $(P S)_{c}$-sequence. For the convenience of the readers we sketch its proof.

THEOREM 1. Let $\left\{u_{n}\right\}$ be a $(P S)_{c}$-sequence in $H_{0}^{1}\left(\Omega_{r}\right)$ for $F_{\Omega_{r}}$. Then there are a nonnegative integer $k, k$ sequences $\left\{y_{n}^{i}\right\}$ of points of the form $\left(x_{n}^{\prime}, m_{n}+1 / 2\right)$ for integers $m_{n}, i=1,2, \cdots, k, u^{0}$ in $H_{0}^{1}\left(\Omega_{r}\right)$ solving equation $\left(1_{\Omega_{r}}\right)$ and nontrivial functions $u^{1}, \cdots, u^{k}$ in $H^{1}\left(\mathbf{R}^{N}\right)$ solving equation $\left(1_{\mathbf{R}^{N}}\right)$. Moreover there is a subsequence $\left\{u_{n}\right\}$ satisfying

(1) $u_{n}(x)=u^{0}(x)+u^{1}\left(x-x_{n}^{1}\right)+\cdots+u^{k}\left(x-x_{n}^{k}\right)+o(1)$ strongly, where $x_{n}^{i}=y_{n}^{1}+\cdots+y_{n}^{i} \rightarrow \infty, i=1,2, \cdots, k$.

(2) $\left\|u_{n}\right\|_{\Omega_{r}}^{2}=\left\|u^{0}\right\|_{\Omega_{r}}^{2}+\left\|u^{1}\right\|^{2}+\cdots+\left\|u^{k}\right\|^{2}+o(1)$,

(3) $\quad F_{\Omega_{r}}\left(u_{n}\right)=F_{n_{r}}\left(u^{0}\right)+F\left(u^{1}\right)+\cdots+F\left(u^{k}\right)+o(1)$.

If $u_{n} \geqslant 0$ for $n=1,2, \cdots$, then $u^{1}, \cdots, u^{k}$ can be chosen as positive solutions, and $u^{0} \geqslant 0$.

Proof: Note that each function in $H_{0}^{1}\left(\Omega_{r}\right)$, by extending it to be 0 outside $\Omega_{r}$, can be considered as a function in $H^{1}\left(\mathrm{R}^{N}\right)$. Since

$$
\begin{aligned}
& F_{\Omega_{r}}\left(u_{n}\right)=\frac{1}{2}\left\|u_{n}\right\|_{\Omega_{r}}^{2}-\frac{1}{p}\left\|u_{n}\right\|_{L^{p}\left(\Omega_{r}\right)}^{p}=c+o(1), \\
& F_{\Omega_{r}}^{\prime}\left(u_{n}\right)=\left\|u_{n}\right\|_{\Omega_{r}}^{2}-\left\|u_{n}\right\|_{L^{p}\left(\Omega_{r}\right)}^{p}=o\left(\left\|u_{n}\right\|_{\Omega_{r}}\right),
\end{aligned}
$$

we see that $\left\{u_{n}\right\}$ is bounded in $H_{0}^{1}\left(\Omega_{r}\right)$. Take a subsequence $\left\{u_{n}\right\}$ and $u^{0}$ in $H_{0}^{1}\left(\Omega_{r}\right)$ such that $u_{n} \rightarrow u^{0}$ weakly in $H_{0}^{1}\left(\Omega_{r}\right)$, almost everywhere in $\Omega_{r}$, and strongly in $L_{\text {loc }}^{p}\left(\Omega_{r}\right)$. Let $\varphi_{n}^{1}=u_{n}-u^{0}$. By the Brezis-Lieb Lemma (see [5]) and the Vitali 
Lemma, we have

$$
\begin{gathered}
-\Delta u^{0}+\lambda u^{0}=\left|u^{0}\right|^{p-2} u^{0} \text { in } \Omega_{r} \\
\left\|\varphi_{n}^{1}\right\|_{\Omega_{r}}^{2}=\left\|u_{n}\right\|_{\Omega_{r}}^{2}-\left\|u^{0}\right\|_{\Omega_{r}}^{2}+o(1) \\
\left\|\varphi_{n}^{1}\right\|_{L^{p}\left(\Omega_{r}\right)}^{p}=\left\|u_{n}\right\|_{L^{p}\left(\Omega_{r}\right)}^{p}-\left\|u^{0}\right\|_{L^{p}\left(\Omega_{r}\right)}^{p}+o(1) . \\
F_{\Omega_{r}}\left(\varphi_{n}^{1}\right)=F_{\Omega_{r}}\left(u_{n}\right)-F_{\Omega_{r}}\left(u^{0}\right)+o(1) \\
F_{\Omega_{r}}^{\prime}\left(\varphi_{n}^{1}\right)=o(1) \quad \text { strongly. }
\end{gathered}
$$

CASE 1. If $\varphi_{n}^{1} \rightarrow 0$ strongly, then

$$
\begin{gathered}
u_{n}(x)=u^{0}(x)+o(1) \quad \text { strongly } \\
\left\|u_{n}\right\|_{\Omega_{r}}^{2}=\left\|u^{0}\right\|_{\Omega_{r}}^{2}+o(1) \\
F_{\Omega_{r}}\left(u_{n}\right)=F_{\Omega_{r}}\left(u^{0}\right)+o(1)
\end{gathered}
$$

In order to prove the second case, we need the following lemma in which the proof follows from Bahri-Lions [2]:

Decompose $\mathbf{R}^{N}$ into nonoverlapping countable cubes $Q_{i}$ with centres $\left(x^{\prime}, m+1 / 2\right)$ for integers $m$ and side length 1 . Define the concentration function $h_{k}$ of $\left|u_{k}\right|^{2}$ by

$$
h_{k}=\sup _{|i|=0,1,2, \ldots} \int_{Q_{i}}\left|u_{k}\right|^{2}
$$

LEMMA 2. If $\left\{u_{k}\right\}$ is a bounded $(P S)_{c}$ sequence in $H^{1}\left(\mathbf{R}^{N}\right)$ such that $h_{k} \rightarrow 0$ as $k \rightarrow \infty$, then $u_{k} \rightarrow 0$ strongly in $H^{1}\left(\mathbf{R}^{N}\right)$.

Proof: For $2<q<r<2^{*}=2 N /(N-2), q=(1-t) \cdot 2+t r, t>0, s=t r / 2 \geqslant$ 1. Now

$$
\begin{aligned}
\int_{R^{N}}\left|u_{k}\right|^{q} & =\sum_{i} \int_{Q_{i}}\left|u_{k}\right|^{(1-t) \cdot 2}\left|u_{k}\right|^{t r} \\
& \leqslant \sum_{i}\left(\int_{Q_{i}}\left|u_{k}\right|^{2}\right)^{(1-t)}\left(\int_{Q_{i}}\left|u_{k}\right|^{r}\right)^{t} \\
& \leqslant\left(h_{k}\right)^{(1-t)} \sum_{i}\left(\int_{Q_{i}}\left|u_{k}\right|^{r}\right)^{t} \\
& \leqslant c\left(h_{k}\right)^{(1-t)} \sum_{i}\left(\int_{Q_{i}}\left|\nabla u_{k}\right|^{2}+u_{k}^{2}\right)^{t r / 2} \\
& \leqslant c\left(h_{k}\right)^{1-t}\left[\sum_{i} \int_{Q_{i}}\left(\left|\nabla u_{k}\right|^{2}+u_{k}^{2}\right)\right]^{t r / 2} \\
& \leqslant c\left(h_{k}\right)^{1-t}\left(\left\|u_{k}\right\|_{H^{1}\left(R^{N}\right)}\right)^{(t r) / 2} \\
& \leqslant c\left(h_{k}\right)^{1-t}=o(1) \text { as } k \rightarrow \infty .
\end{aligned}
$$


By the $(P S)_{c}$ condition, we have

$$
\left\|u_{k}\right\|_{H^{1}\left(R^{N}\right)}^{2}-\int_{R^{N}}\left|u_{k}\right|^{p+1}=\varepsilon_{k}\left\|u_{k}\right\|_{H^{1}\left(R^{N}\right)}=o(1)
$$

where $\varepsilon_{k}=o(1)$. Since $\int_{R^{N}}\left|u_{k}\right|^{k+1}=o(1)$, we have

$$
\left\|u_{k}\right\|_{H^{1}\left(R^{N}\right)}=o(1), \quad \text { as } \quad k \rightarrow \infty
$$

This completes the proof.

CASE 2. If $\varphi_{n}^{1}$ does not converge to 0 strongly, then by Lemma 2 there is a subsequence $\left\{\varphi_{n}^{1}\right\}$ and $\delta>0$ such that

$$
\sup _{|i|=0,1,2, \ldots} \int_{Q_{i}}\left|u_{k}\right|^{2} \geqslant \delta \text { for } \quad n=1,2, \cdots
$$

where $\left\{Q_{i}\right\}$ are as in Lemma 2. For each $n$, find a $Q_{n}^{1}$ with centre $y_{n}^{1}$ of the form $\left(x_{n}^{\prime}, m_{n}+1 / 2\right)$ such that

$$
\left\|\varphi_{n}^{1}\right\|_{L^{2}\left(Q_{n}^{1}\right)}^{2} \geqslant \frac{\delta}{2}
$$

Take $u^{1}$ in $H^{1}\left(\mathbf{R}^{N}\right)$ and a subsequence $\left\{\varphi_{n}^{1}\left(x+y_{n}^{1}\right)\right\}$ satisfying $\varphi_{n}^{1}\left(x+y_{n}^{1}\right)-u^{1}(x)$ weakly in $H^{1}\left(\mathbf{R}^{N}\right)$, almost everywhere in $\mathbf{R}^{N}$ and strongly in $L_{l o c}^{p}\left(\mathbf{R}^{N}\right)$. Since

$$
\left\|u^{1}\right\|_{L^{2}(Q)}^{2}=\lim _{n \rightarrow \infty}\left\|\varphi_{n}^{1}\left(x+y_{n}^{1}\right)\right\|_{L^{2}(Q)}^{2} \geqslant \frac{\delta}{2}
$$

where $Q=\left\{\left(x^{\prime}, x_{N}\right) \in \mathbf{R}^{N-1} \times \mathbf{R}|| x^{\prime} \mid<1 / 2,-1 / 2<x_{N}<1 / 2\right\}$, we have $u^{1} \not \equiv 0$.

Let $\varphi_{n}^{2}(x)=\varphi_{n}^{1}\left(x+y_{n}^{1}\right)-u^{1}(x)$. Then $\varphi_{n}^{2}-0$ weakly in $H^{1}\left(\mathbf{R}^{N}\right)$, almost everywhere in $\mathbf{R}^{N}$ and strongly in $L_{l o c}^{p}\left(\mathbf{R}^{N}\right)$. We obtain that $u^{1}$ solves $\left(1_{\mathbf{R}^{N}}\right)$ and satisfies

$$
\left\|u^{1}\right\|^{2} \geqslant \alpha^{p /(p-2)}
$$

and similar equalities as in Case 1 above. Continuing this process, by (2-1), we have to stop after a finite number of steps. This completes the proof.

Let $\left\{u_{n}\right\} \subset M_{\Omega_{r}}$ satisfy $f_{\Omega_{r}}\left(u_{n}\right)=c+o(1)$. Set $v_{n}=c^{1 /(p-2)} u_{n}$ for $n=1,2, \cdots$. Then we have

$$
\begin{aligned}
& F_{\Omega_{r}}\left(v_{n}\right)=\left(\frac{1}{2}-\frac{1}{p}\right) c^{p /(p-2)}+o(1) \\
& F_{\Omega_{r}}^{\prime}\left(v_{n}\right)=o(1) \quad \text { strongly. }
\end{aligned}
$$


CoRollary 3. Let $\left\{u_{n}\right\} \subset M_{\Omega_{r}}$ satisfy $u_{n} \geqslant 0, f_{\Omega_{r}}\left(u_{n}\right)=c+o(1)$ and $\alpha<c<2^{(p-2) / p} \alpha$. Then $\left\{u_{n}\right\}$ contains a strongly convergent subsequence.

PROOF: Set $v_{n}=c^{1 /(p-2)} u_{n}$ for $n=1,2, \cdots$. Then

$$
\begin{aligned}
& F_{\Omega_{r}}\left(v_{n}\right)=\left(\frac{1}{2}-\frac{1}{p}\right) c^{p /(p-2)}+o(1) \\
& F_{n_{r}}^{\prime}\left(v_{n}\right)=o(1) \quad \text { strongly. }
\end{aligned}
$$

By applying Theorem 1 we obtain solutions $v^{0}$ of equation $\left(1_{n_{r}}\right)$ and positive solutions, $v^{1}, \cdots, v^{k}$ of equation $\left(1_{\mathbf{R}^{N}}\right)$ and $\left\{x_{n}^{i}\right\}_{n=1}^{\infty}$ of the form $\left(x_{n}^{\prime}, m_{n}+1 / 2\right), m_{n}$ integers, $i=1, \cdots, n$ such that

$$
\begin{gathered}
v_{n}(x)=v^{0}(x)+v^{1}\left(x-x_{n}^{1}\right)+\cdots+v^{k}\left(x-x_{n}^{k}\right)+o(1) \quad \text { strongly } \\
\left\|v_{n}\right\|_{\Omega_{r}}^{2}=\left\|v^{0}\right\|_{\Omega_{r}}^{2}+\left\|v^{1}\right\|^{2}+\cdots+\left\|v^{k}\right\|^{2}+o(1) \\
F_{\Omega_{r}}\left(v_{n}\right)=F_{\Omega_{r}}\left(v^{0}\right)+F\left(v^{1}\right)+\cdots+F\left(v^{k}\right)+o(1)
\end{gathered}
$$

Note that if $v^{i} \geqslant 0, v^{i} \not \equiv 0, i=1,2, \cdots, k$, then we can take $v^{i}>0, v^{i}$ is unique up to a translation and $F\left(v^{i}\right)=(1 / 2-1 / p) \alpha^{p /(p-2)}$ for $i=1,2, \cdots, k$. Therefore, by $(2-2)$ and (2-3),

$$
\left(\frac{1}{2}-\frac{1}{p}\right) c^{p /(p-2)}=F_{\Omega_{r}}\left(v^{0}\right)+k\left(\frac{1}{2}-\frac{1}{p}\right) \alpha^{p /(p-2)}+o(1) .
$$

If $v^{0} \not \equiv 0$, then $v^{0}>0$ and $F_{\Omega}\left(v^{0}\right)>\left(1 / 2-\frac{1}{p}\right) \alpha^{p /(p-1)}$ by Proposition 5 below. If $\alpha<c<2^{(p-2) / p} \alpha$, then $k=0, v^{0}>0$ and

$$
v_{n}(x)=v^{0}(x)+o(1)
$$

or

$$
u_{n}(x)=u^{0}(x)+o(1)
$$

where $u^{0}=c^{-1 /(p-2)} v^{0}$. Therefore $\left\{u_{n}\right\}$ contains a strongly convergent subsequence.

Take $\xi \in C^{\infty}\left(\mathbf{R}^{+}, \mathbf{R}\right), \eta \in C^{\infty}(\mathbf{R}, \mathbf{R})$ such that

$$
\begin{aligned}
& \xi(t)= \begin{cases}0 & 0 \leqslant t \leqslant \rho \\
1 & t \geqslant 2 \rho\end{cases} \\
& \eta(t)= \begin{cases}0 & t \leqslant 0 \\
1 & t \geqslant 1\end{cases} \\
& 0 \leqslant \xi \leqslant 1, \quad 0 \leqslant \eta \leqslant 1
\end{aligned}
$$


Then $\varphi_{y} \in H_{0}^{1}\left(\Omega_{r}\right)$ and $\int_{\Omega_{r}}\left|\varphi_{r}\right|^{p}=1$. Furthermore we have

LEMma 4. Let $y=\left(y^{\prime}, y_{N}\right)$, then

(1) $\left\|f_{y}-\bar{u}(\cdot-y)\right\|_{L^{p}\left(\mathbf{R}^{N}\right)}=o(1)$ as $\left|y-a_{r}\right| \rightarrow \infty$ and $y_{N} \rightarrow \infty$, or $\rho \rightarrow 0$ and $y_{N} \rightarrow \infty$

(2) $\left\|f_{y}-\bar{u}(\cdot-y)\right\|=o(1)$ as $\left|y-a_{r}\right| \rightarrow \infty$ and $y_{N} \rightarrow \infty$ or $\rho \rightarrow 0$ and $y_{N} \rightarrow \infty$

ProOF: (1)

$$
\begin{aligned}
& \left\|f_{y}(x)-\bar{u}(x-y)\right\|_{L^{p}\left(\mathbf{R}^{N}\right)}^{p} \\
& \quad=\int_{\mathbb{R}^{N}}\left|\xi\left(\left|x-a_{r}\right|\right) \eta\left(x_{N}\right)-1\right|^{p}|\bar{u}(x-y)|^{p} d x \\
& \quad \leqslant 2^{p} \int_{B_{2 \rho}\left(a_{r}\right) \cup\left\{x_{N} \leqslant 1\right\}}|\bar{u}(x-y)|^{p} d x \\
& \quad=o(1) \text { as }\left|y-a_{r}\right| \rightarrow \infty \text { and } y_{N} \rightarrow \infty, \text { or } \rho \rightarrow 0 \text { and } y_{N} \rightarrow \infty .
\end{aligned}
$$

(2)

$$
\begin{aligned}
& \left\|f_{y}(x)-\bar{u}(x-y)\right\|^{2} \\
& \quad=\left\|\left(\xi\left(\left|x-x_{r}\right|\right) \eta\left(x_{N}\right)-1\right) \bar{u}(x-y)\right\|^{2} \\
& \quad \leqslant \frac{c}{\rho} \int_{B_{2 \rho}\left(a_{r}\right) \cup\left\{x_{N} \leqslant 1\right\}}\left(|\nabla \bar{u}(x-y)|^{2}+|\bar{u}(x-y)|^{2}\right) \\
& \quad=o(1) \text { as }\left|y-a_{r}\right| \rightarrow \infty \text { and } y_{N} \rightarrow \infty, \text { or } \rho \rightarrow 0 \text { and } y_{N} \rightarrow \infty .
\end{aligned}
$$

PROPOSITION 5. Equation $\left(1_{n_{r}}\right)$ does not have any ground state solution.

Proof: Note that $\alpha_{\Omega_{r}} \geqslant \alpha$ since each function in $H_{0}^{1}(\Omega)$ can be extended by 0 outside $\Omega_{r}$. Take a sequence $\left\{y^{n}\right\}$ in $\Omega_{r}$ such that

$$
\left|y^{n}-a_{r}\right| \rightarrow \infty \text { and } y_{N}^{n} \rightarrow \infty \text { as } n \rightarrow \infty .
$$

Then, by Lemma 4,

$$
\begin{aligned}
& \left\|f_{y^{n}}-\bar{u}\left(\cdot-y^{n}\right)\right\|_{L^{p}\left(\mathbf{R}^{N}\right)}=o(1) \quad \text { as } \quad n \rightarrow \infty \\
& \left\|f_{y^{n}}-\bar{u}\left(\cdot-y^{n}\right)\right\|=o(1) \text { as } n \rightarrow \infty \text {. }
\end{aligned}
$$

Thus $\left\{\varphi_{y^{n}}\right\} \subset H_{0}^{1}(\Omega)$ is such that

$$
\begin{gathered}
\int_{\Omega_{r}}\left|\varphi_{y^{n}}\right|^{p}=1 \text { for } n=1,2, \cdots \\
\left\|\varphi_{y^{n}}\right\|^{2} \longrightarrow \alpha
\end{gathered}
$$

or $\alpha_{\Omega_{r}} \leqslant \alpha$. We then conclude that $\alpha_{\Omega_{r}}=\alpha$. By the maximum principle, there does not exist any ground state solution of equation $\left(1_{\Omega_{r}}\right)$. In other words, if $u$ is a solution of equation $\left(1_{\Omega_{r}}\right)$ satisfying $\int_{\Omega_{r}}|u|^{p}=1$, then $\|u\|_{\Omega_{r}}^{2}>\alpha$. 
REMARK 6. By Lemma 4(1), there is $r_{1}>0$ such that

$$
\frac{1}{2} \leqslant\left\|f_{y}\right\|_{L P\left(\mathrm{n}_{r}\right)} \leqslant \frac{3}{2}
$$

where $r \geqslant r_{1}$ and $\left|y-a_{r}\right| \geqslant r / 2$ and $y_{N} \geqslant r / 2$.

Set

$$
\chi(t)= \begin{cases}1 & \text { if } 0 \leqslant t \leqslant 1 \\ \frac{1}{t} & \text { if } 1 \leqslant t<\infty\end{cases}
$$

and define $\beta: H^{1}\left(\mathbf{R}^{N}\right) \rightarrow \mathbf{R}^{N}$ by

$$
\beta(u)=\int_{\mathbf{R}^{N}} u^{2}(x) \chi(|x|) x d x
$$

For $r \geqslant r_{1}$, let

$$
\begin{aligned}
& V_{r}=\left\{\left.u \in H_{0}^{1}\left(\Omega_{r}\right)\left|\int_{\Omega_{r}}\right| u\right|^{p}=1, \beta(u)=a_{r}\right\}, \\
& c_{r}=\inf _{u \in V_{r}}\|u\|_{\Omega_{r}}^{2} .
\end{aligned}
$$

Then we have:

LEMMA $7 . c_{r}>\alpha$.

PROOF: It is easy to see that $c_{r} \geqslant \alpha$. Suppose $c_{r}=\alpha$. Take a sequence $\left\{v_{m}\right\} \subset$ $H_{0}^{1}\left(\Omega_{r}\right)$ such that

$$
\begin{aligned}
\left\|v_{m}\right\|_{L^{p}\left(\Omega_{r}\right)} & =1, \beta\left(v_{m}\right)=a_{r} \quad \text { for } \quad m=1,2, \cdots, \\
\left\|v_{m}\right\|_{\Omega_{r}}^{2} & =\alpha+o(1) .
\end{aligned}
$$

Let $u_{m}=\alpha^{1 /(p-2)} v_{m}$ for $m=1,2, \cdots$. Then

$$
\begin{aligned}
& F_{\Omega_{r}}\left(u_{m}\right)=\left(\frac{1}{2}-\frac{1}{p}\right) \alpha^{p /(p-2)}+o(1) \\
& F_{\Omega_{r}}^{\prime}\left(u_{m}\right)=o(1) \text { strongly. }
\end{aligned}
$$

By the maximum principle, $\left\{u_{m}\right\}$ does not contain any convergent subsequence. By Theorem 1 , there is a sequence $\left\{x_{m}\right\}$ of the form $\left(x_{m}^{\prime}, m+\frac{1}{2}\right)$ for integers $m$ such that

$$
\begin{gathered}
\left|x_{m}\right| \longrightarrow \infty \\
u_{m}(x)=\bar{u}\left(x-x_{m}\right)+o(1) \quad \text { strongly. }
\end{gathered}
$$


Since $\bar{u}$ is radially symmetric, we may take $m$ to be positive. We may assume that $\left|x_{m}\right| \geqslant 4$ from $m=1,2, \cdots$. Now

$$
\begin{aligned}
\left\langle\beta\left(\bar{u}\left(x-x_{m}\right)\right), x_{m}\right\rangle= & \int_{\mathbf{R}^{N}} \bar{u}^{2}\left(x-x_{m}\right) \chi(|x|)\left\langle x, x_{m}\right\rangle d x \\
= & \int_{\mathbf{R}_{+}^{N}} \bar{u}^{2}\left(x-x_{m}\right) \chi(|x|)\left\langle x, x_{m}\right\rangle d x \\
& +\int_{\left(\mathbf{R}_{-}^{N}\right)} \bar{u}^{2}\left(x-x_{m}\right) \chi(|x|)\left\langle x, x_{m}\right) d x \\
\geqslant & \int_{B_{1}\left(x_{m}\right)} \bar{u}^{2}\left(x-x_{m}\right) \chi(|x|)\left\langle x, x_{m}\right\rangle d x \\
& +\int_{\mathbf{R}_{-}^{N}} \bar{u}^{2}\left(x-x_{m}\right) \chi(|x|)\left\langle x, x_{m}\right\rangle d x
\end{aligned}
$$

Note that there are $c_{1}>0, c_{2}>0$ such that for $x \in B_{1}\left(x_{m}\right)$, we have

$$
\begin{aligned}
\bar{u}^{2}\left(x-x_{m}\right) & \geqslant c_{1}, \\
\left\langle x, x_{m}\right\rangle & \geqslant c_{2}|x|\left|x_{m}\right| \text { for } m=1,2, \cdots .
\end{aligned}
$$

Thus

$$
\begin{aligned}
\int_{B_{1}\left(x_{m}\right)} \bar{u}^{2}\left(x-x_{m}\right) \chi(|x|)\left\langle x, x_{m}\right\rangle d x & \geqslant c_{1} c_{2} \int_{B_{1}\left(x_{m}\right)} \chi(|x|)|x|\left|x_{m}\right| d x \\
& \geqslant c_{3}\left|x_{m}\right|^{N+1}, \quad c_{3}>0 \text { a constant. }
\end{aligned}
$$

Next, for $0 . \leqslant s<\infty$, by $(1-1)$,

$$
\bar{u}(s) s^{(N-1) / 2} e^{\sqrt{\lambda} s} \leqslant c_{4} \text { for } \quad c_{4}>0 .
$$

Now

$$
\begin{aligned}
\int_{\mathbf{R}_{-}^{N}} \bar{u}^{2}\left(x-x_{m}\right) \chi(|x|)\left\langle x, x_{m}\right) d x & \leqslant c_{4}^{2} \int_{\mathbf{R}_{-}^{N}} \frac{\chi(|x|)|x|\left|x_{m}\right|}{\left|x-x_{m}\right|^{(N-1)} e^{2 \sqrt{\lambda}\left|x-x_{m}\right|}} \\
& \leqslant \frac{c_{5}}{e^{\sqrt{\lambda}\left|x_{m}\right|}}, \quad c_{5}>0 \quad \text { a constant. }
\end{aligned}
$$

Therefore

$$
\left\langle\beta\left(\bar{u}\left(x-x_{m}\right)\right), x_{m}\right\rangle \geqslant c_{3}\left|x_{m}\right|^{N+1}-\frac{c_{5}}{e^{\sqrt{\lambda}\left|x_{m}\right|}},
$$

or

$$
\left\langle\beta\left(\bar{u}\left(x-x_{m}\right)\right), \frac{x_{m}}{\left|x_{m}\right|}\right\rangle \geqslant c_{3}\left|x_{m}\right|^{N}-\frac{c_{5}}{\left|x_{m}\right| e^{\sqrt{\lambda}\left|x_{m}\right|}} .
$$


We conclude that

$$
\begin{aligned}
\alpha^{1 /(p-2)}\left|a_{r}\right| & \geqslant\left\langle\beta\left(u_{m}\right), \frac{x_{m}}{\left|x_{m}\right|}\right\rangle \\
& =\left\langle\beta\left(\bar{u}\left(x-x_{m}\right)\right), \frac{x_{m}}{\left|x_{m}\right|}\right\rangle+o(1) \\
& \geqslant c_{3}\left|x_{m}\right|^{N}+o(1),
\end{aligned}
$$

a contradiction. Thus $c_{\boldsymbol{r}}>\alpha$.

REMARK 8. By Lemma 4 (2), there is $r_{2} \geqslant r_{1}$ such that

$$
\alpha<\left\|\varphi_{y}\right\|^{2}<\frac{c_{r}+\alpha}{2}
$$

where $r \geqslant r_{2}$ and $\left|y-a_{r}\right| \geqslant r / 2$ and $y_{N} \geqslant r / 2$.

LEMMA 9. There is $r_{3} \geqslant r_{2}$ such that if $r \geqslant r_{3}$, then

$$
\left\langle\beta\left(\varphi_{y}\right), y\right\rangle>0 \quad \text { for } \quad y \in \partial\left(B_{r / 2}\left(a_{r}\right)\right)
$$

PROOF: By (2-4), $2 / 3 \leqslant c_{y} \leqslant 2$. For $r \geqslant r_{2}$, let

$$
\begin{gathered}
A_{\left((3 / 8) r_{,}(5 / 8) r\right)}=\left\{x \in \mathbf{R}^{N}\left|\frac{3}{8} r \leqslant\right| x-a_{r} \mid \leqslant \frac{5}{8} r\right\} \\
\mathbf{R}_{+}^{N}(y)=\left\{x \in \mathbf{R}^{N} \mid\langle x, y\rangle>0\right\}, \\
\mathbf{R}_{-}^{N}(y)=\left\{x \in \mathbf{R}^{N} \mid\langle x, y\rangle<0\right\} . \\
\left\langle\beta\left(\varphi_{y}\right), y\right\rangle=c_{y}\left[\int_{\mathbf{R}_{+}^{N}(y)} \xi^{2}\left(\left|x-a_{r}\right|\right) \eta^{2}\left(x_{N}\right) \bar{u}^{2}(x-y) \chi(|x|)\langle x, y\rangle d x\right. \\
\left.+\int_{\mathbf{R}_{-}^{N}(y)} \xi^{2}\left(\left|x-a_{r}\right|\right) \eta^{2}(x) \bar{u}^{2}(x-y) \chi(|x|)\langle x, y\rangle d x\right] \\
\geqslant \frac{2}{3}\left[\int_{A_{((3 / 8) r,(5 / 8) r)}} \bar{u}^{2}(x-y) \chi(|x|)\langle x, y\rangle\right. \\
+\int_{\mathbf{R}_{-}^{N}(y)} \bar{u}^{2}(x-y) \chi(|x|)\langle x, y\rangle d x .
\end{gathered}
$$


Now

$$
\begin{aligned}
\int_{A((3 / 8) r,(5 / 8) r)} \bar{u}^{2}(x-y) \chi(|x|)\langle x, y\rangle d x & \geqslant c_{6} \int_{A\left((3 / 8) r_{,}(5 / 8) r\right)} \chi(|x|)|x||y| d x \text { for } c_{8}>0 \\
& \geqslant c_{6}|y|\left[\left(\frac{5}{8} r\right)^{N}-\left(\frac{3}{8} r\right)^{N}\right] \\
& \geqslant c_{7} r^{N+1} \text { for } c_{7}>0 . \\
\int_{\mathbf{R}_{-}^{N}(y)} \bar{u}^{2}(x-y) \chi(|x|)\langle x, y\rangle d x & \leqslant c_{8} \int_{\mathbf{R}_{-}^{N}(y)} \frac{|y|}{|x-y|^{(N-1)} e^{2 \sqrt{\lambda}|x-y|} d x \text { for } c_{8}>0} \\
& \leqslant c_{9} \frac{1}{e^{\sqrt{\lambda} r}} \text { for } c_{9}>0 .
\end{aligned}
$$

Therefore, there is $r_{3} \geqslant r_{2}$, such that if $r \geqslant r_{3},\left|y-a_{r}\right|=r / 2$

$$
\left\langle\beta\left(\varphi_{y}\right), y\right\rangle \geqslant c_{7} r^{N+1}-c_{8} \frac{1}{e \sqrt{\lambda} r}>0 .
$$

This completes the proof.

By Lemma 4 and Lemma 9 , fix $\rho_{0}>0, r_{0} \geqslant r_{3}$ such that if $0<\rho \leqslant \rho_{0}, r \geqslant r_{0}$, then $\left\|\varphi_{y}\right\|_{\Omega_{r}}^{2}<2^{(p-2) / p_{\alpha}}$ for $y \in \overline{B_{r / 2}}\left(a_{r}\right)$. From now on, fix $\rho_{0}, r_{0}$, for $r \geqslant r_{0}$. Let

$$
\begin{aligned}
& B=\left\{\varphi_{y}|| y-a_{r} \mid \leqslant \frac{r}{2}\right\}, \\
& \Gamma=\left\{h \in C\left(V_{r}, V_{r}\right) \mid h(u)=u \text { if }\|u\|_{\Omega_{r}}^{2}<\frac{c_{r}+\alpha}{2}\right\} .
\end{aligned}
$$

Lemma 10. $h(B) \cap V_{r} \neq \emptyset$ for each $h \in \Gamma$.

Proof: Let $h \in \Gamma$ and $H(x)=\beta \circ h \circ \varphi_{x}: \mathbf{R}^{N} \rightarrow \mathbf{R}^{N}$. Consider the homotopy, for $0 \leqslant t \leqslant 1$,

$$
F(t, x)=(1-t) H(x)+t I(x) \quad \text { for } \quad x \in \mathbf{R}^{N} .
$$

If $x \in \partial\left(B_{r / 2}\left(a_{r}\right)\right)$, then, by Remark 8 and Lemma 9 ,

$$
\begin{gathered}
\left\langle\beta\left(\varphi_{x}\right), x\right\rangle>0, \\
\alpha<\left\|\varphi_{x}\right\|^{2}<\frac{c_{r}+\alpha}{2} .
\end{gathered}
$$

Then

$$
\begin{aligned}
\langle F(t, x), x\rangle & =\langle(1-t) H(x), x\rangle+\langle t x, x\rangle \\
& =(1-t)\left\langle\beta\left(\varphi_{x}\right), x\right\rangle+t\langle x, x\rangle \\
& >0
\end{aligned}
$$


Thus $F(t, x) \neq 0$ for $x \in \partial\left(B_{r / 2}\left(a_{r}\right)\right)$. By the homotopic invariance of the degree

$$
d\left(H(x), B_{r / 2}\left(a_{r}\right), a_{r}\right)=d\left(I, B_{r / 2}\left(a_{r}\right), a_{r}\right)=1
$$

There is $x \in B_{r / 2}\left(a_{r}\right)$ such that

$$
a_{r}=H(x)=\beta\left(h \circ \varphi_{x}\right)
$$

Thus $h(B) \cap V_{r} \neq \emptyset$ for each $h \in \Gamma$.

Now we are in the position to prove Theorem A: Consider the class of mappings

$$
F=\left\{h \in C\left(\overline{B_{r / 2}\left(a_{r}\right)}\right), H^{1}\left(R_{N}\right):\left.h\right|_{\partial B_{r / 2}\left(a_{r}\right)}=\varphi_{y}\right\}
$$

and set

$$
c=\inf _{h \in F} \sup _{y \in \frac{B_{r / 2}\left(a_{r}\right)}{}}\|h(y)\|_{n_{r}}^{2}
$$

It follows from Lemmas 4-10, with the appropriate choice of $r$ that

$$
\alpha<c_{r}=\inf _{u \in V_{\gamma}}\|u\|_{\Omega_{r}}^{2} \leqslant c<2^{(p-2) / p} \alpha
$$

and

$$
\max _{\delta B_{r / 2}\left(a_{r}\right)}\|h(y)\|_{\Omega_{r}}^{2}<\max _{B_{r / 2}\left(a_{r}\right)}\|h(y)\|_{\Omega_{r}}^{2}
$$

Theorem A then follows by applying the version of the mountain pass theorem from Brezis-Nirenberg [6].

\section{REFERENCES}

[1] J. Ai and X.P. Zhu, 'Positive solutions of inhomogeneous elliptic boundary value problems in the half space', Comm. Partial Differential Equations 15 (1990), 1421-1446.

[2] A. Bahri and P.L. Lions, 'On the existence of a positive solution of semilinear elliptic equations in unbounded domains', (preprint).

[3] V. Benci and G. Cerami, 'Positive solutions of some nonlinear elliptic problems in exterior domains', Arch. Rational Mech. Anal. 99 (1987), 283-300.

[4] H. Berestycki and P.L. Lions, 'Nonlinear scalar field equations, I., existence of ground state', Arch. Rational Mech. Anal. 82 (1983), 313-345.

[5] H. Brezis and E. Lieb, 'A relation between pointwise convergence of functions and convergence of functionals', Proc. Amer. Math. Soc. 88 (1983), 486-490.

[6] H. Brezis and L. Nirenberg, 'Remarks on finding critical points', Comm. Pure Appl. Maths. 44 (1991), 939-963. 
[7] J.M. Coron, 'Topologie et cas limite des injections de Sobolev', C. R. Acad. Sci. Paris Ser. 1299 (1984), 55-64.

[8] M.J. Esteban and P.L. Lions, 'Existence and non-existence results for semilinear elliptic problems in unbounded domains', Proc. Royal Soc. Edinburgh Sect. A 93 (1982), 1-14.

[9] B. Gidas, W.M. Ni and L. Nirenberg, 'Symmetry of positive solutions of nonlinear elliptic equations in $\mathrm{R}^{N}$, Adv. Math. 7 (1981), 369-402.

[10] M. Grossi, 'Multiplicity results for semilinear equations with lack of compactness', $D$ ifferential Integral Equations 6 (1993), 807-823.

[11] M.K. Kwong, 'Uniqueness of positive solutions of $\Delta u-u+u^{p}=0$ in $\mathbf{R}^{N}$, Arch. Rational Mech. Anal. 105 (1989), 243-666.

[12] W.C. Lien, S.Y. Tzeng and H.C. Wang, 'Existence of solutions of semilinear elliptic problems on unbounded domains', Differential Integral Equations 6 (1993), 1281-1298.

Department of Mathematics

National Tsing Hua University

Hsinchu

Taiwan

e-mail: hwang@math.nthu.edu.tw 